\title{
Growth of two-dimensional arrays of uncapped gold nanoparticles on silicon substrates
}

\author{
ANINDYA DAS, SOMA DAS and A K RAYCHAUDHURI* \\ DST Unit for Nanoscience, S.N. Bose National Centre for Basic Sciences, Kolkata 700 098, India
}

\begin{abstract}
A method of preparing large area patterned 2D arrays of uncapped gold (Au) nanoparticles has been developed. The pattern has been formed using self-assembly of uncapped Au nanoparticles. The Au nanoparticles were synthesized via toluene/water two phase systems using a reducing agent and colloidal solution of Au nanoparticles was produced. These nanoparticles have been prepared without using any kind of capping agent. Analysis by TEM showed discrete Au nanoparticles of $4 \mathbf{n m}$ average diameter. AFM analysis also showed similar result. The TEM studies showed that these nanoparticles formed self-assembled coherent patterns with dimensions exceeding $500 \mathrm{~nm}$. Spin coating on silicon substrate by suitably adjusting the speed can self-assemble these nanoparticles to lengths exceeding $1 \mu \mathrm{m}$.
\end{abstract}

Keywords. Gold nanoparticles; 2D array; spin-coating; self-assembly; TEM; AFM.

\section{Introduction}

Colloidal inorganic nanoparticles have size-dependent optical, optoelectronic and material properties. It is expected that superstructures of these particles can have a range of practical applications. For example, metal nanoparticles can be used as the catalyst to grow nanowires. Transition metal nanoparticles are also useful in fabricating high density magnetic recording media (Hong et al 2002). Besides, nanoparticles possessing surface plasma resonance can also be used as biosensors (Green et al 2000). In the application of nanoparticles, one of the fundamental issues is how to distribute nanoparticles uniformly on the substrate with precise control. There are several methods proposed for dispersing nanoparticles on a substrate. In many studies, the self-assembly technique was employed utilizing the chemical interaction between nanoparticles and the substrate surface. For example, organic molecules containing thiol $(-\mathrm{SH})$ or amine $\left(-\mathrm{NH}_{2}\right)$ can be adsorbed on the gold surface and form a well organized selfassembly monolayer on the substrate (Liu et al 2003). Another popular method is lithography, which is able to produce a well ordered pattern. However, this method requires a lot of processing procedures and is relatively complicated and expensive. Therefore, a simpler and cheaper method by the traditional spin coating has recently been proposed (Cho et al 2002; Hong et al 2002; Choi et al 2003; Kodama et al 2003; Liu et al 2003) as an alternative.

\footnotetext{
*Author for correspondence (arup@bose.res.in)
}

Preparation of highly monodisperse gold nanoparticles through different techniques is still attracting significant interest (Lin et al 2001; Zhou et al 2002), and several methods have been successfully developed and tested (Brust et al 1994; Spatz et al 1996). Reducing metal salts in the presence of a surfactant in a two-phase system and arresting the size of the prepared metal particle by a capping agent already present in the solution, are the most general methods employed for synthesis of monodisperse colloidal particles (Brust et al 1994). Capping agents such as long-chain alkane thiols are usually added to stabilize the organosol. The size, shape, and interparticle separation of these uniform size distribution particles are crucially controlled by the nature of the capping agents (Martin et al 2000; Weare et al 2000).

In this paper, we have done experiments with uncapped $\mathrm{Au}$ nanoparticle and investigated the nature of its selfassembly without any ligand. Without using capping agents the $\mathrm{Au}$ sol was prepared here by controlling the rate of addition of sodium borohydride, stirring speed, temperature and concentration of the reactants. We found that gold nanoparticles formed self-assembled coherent patterns with dimensions more than $500 \mathrm{~nm}$. We were able to arrange the islands of $\mathrm{Au}$ nanoparticles into larger patterns by spin coating on silicon substrate. There were two levels of assembly. In one level the Au nanoparticles with size around $4-5 \mathrm{~nm}$ were self-assembled into islands of sizes in the scale of 30-500 $\mathrm{nm}$. In the second level, these islands were arranged to sizes larger than few $\mu \mathrm{m}$.

In this work, we have shown that large pattern of $\mathrm{Au}$ nanoparticles can be formed by spin-coating. It has also been noted that the patterns form only on the hydrophilic silicon substrates (with native oxide layer on top of it). 


\section{Experimental}

\subsection{Preparation of $\mathrm{Au}$ sol}

$\mathrm{Au}$ sol was prepared by following the procedure (Drake et al 2002) briefly described below. The synthesis process of such small particles does not use any kind of capping agent and it is achieved only by controlling the rate of the reaction. Tetrahexylammonium bromide $(1.74 \mathrm{~g})$ was dissolved in toluene $(80 \mathrm{~mL})$ to produce a $50 \mathrm{mM}$ solution. This was combined with a $30 \mathrm{mM}$ solution of hydrogen tetrachloroaurate $(0.35 \mathrm{~g})$ in distilled water $(30 \mathrm{~mL})$. The suspension was shaken until all the tetrachloroaurate had transferred to the organic phase. A fresh $0.4 \mathrm{M}$ solution of sodium borohydride $(0.39 \mathrm{~g})$ in distilled water $(25 \mathrm{~mL})$ was prepared and added slowly to the toluene phase. On addition, the colour of the toluene phase changed from a clear orange solution to an opaque deep ruby red solution. The lower aqueous phase turned colourless. The mixture was stirred for $30 \mathrm{~min}$ before removal of the two phases. The toluene phase was washed with water and dried over $\mathrm{MgSO}_{4}$. The concentration of $\mathrm{Au}$ nanoparticles in toluene was $2.54 \mathrm{mg} / \mathrm{ml}$. The preparation method is summarized in a flow chart below.

\section{Preparation of Au Sol}

\begin{tabular}{|c|c|}
\hline $\begin{array}{l}\text { Tetrahexylammonium } \\
\text { bromide was dissolved in } \\
\text { toluene }\end{array}$ & $\begin{array}{l}\text { Combined with a solution of } \\
\text { hydrogen tetrachloroaurate in } \\
\text { distilled water }\end{array}$ \\
\hline $\begin{array}{l}\text { Sodium borohydride in } \\
\text { distilled water was added } \\
\text { slowly to the toluene phase }\end{array}$ & $\begin{array}{l}\text { Suspension was shaken until } \\
\text { all the tetrachloroaurate had } \\
\text { transferred to the organic } \\
\text { phase }\end{array}$ \\
\hline $\begin{array}{l}\text { On addition the toluene phase } \\
\text { changed color from a clear } \\
\text { orange solution to an opaque } \\
\text { deep ruby red solution }\end{array}$ & $\begin{array}{l}\text { After separating the two } \\
\text { phases the toluene phase } \\
\text { (Au Sol) was washed with } \\
\text { water }\end{array}$ \\
\hline
\end{tabular}

Briefly, the rate of $\mathrm{NaBH}_{4}$ addition (thereby forming the particle by reduction) and the rate of diffusion into the non-aqueous phase decide the degree of agglomeration. If the rate of addition of the reducing agent is less than the rate at which the particles diffuse into the non-aqueous phase one can avoid agglomeration without adding explicitly any capping agent. When the particles are spin coated on a substrate from toluene, one cannot rule out adsorption of toluene on Au which can be detected by FTIR data.

\section{Results and discussion}

\subsection{Ultra violet-visible spectrum analysis}

Surface plasmon resonance of Au nanoparticles is a good diagnostic tool for formation of the nanoparticles. We have taken a UV-vis spectrum of the Au nanoparticles so formed. The spectra was taken in sol form (with toluene) as well as after coating a glass substrate (with toluene evaporated). A UV-vis spectrum of the prepared Au sol taken in solvent (toluene) is shown in figure 1a. A strong absorption at $\sim 523 \mathrm{~nm}$ was observed, this resonance corresponds to the excitation of surface plasmon vibrations in the Au nanoparticles. This is typical of Au nanoparticles (Underwood and Mulvaney 1994; Maye et al 2000). The absorption spectrum of the multilayered film of $\mathrm{Au}$ nanoparticles coated on glass substrate is shown in figure 1b. In this case the toluene is fully evaporated. The absorption band is shifted to $\sim 592 \mathrm{~nm}$. The exact frequency of the surface plasmon resonance depends on the surrounding dielectric media (Underwood and Mulvaney 1994). This shift is attributed to the difference in the media in which the Au nanoparticles are embedded.

\subsection{Analysis by transmission electron microscope (TEM)}

Analysis by TEM (figure 2a) shows discrete Au nanoparticles. The TEM data were taken on carbon coated grids using a JEOL $(200 \mathrm{keV})$ machine. The average particle size calculated from TEM images is $3.97 \pm 0.45 \mathrm{~nm}$. The particle size distribution is shown in figure $2 b$.

\subsection{Analysis of Au self assembled islands by atomic force microscope (AFM)}

Determination of size of $\mathrm{Au}$ nanoparticles is carried out by TEM by placing them on a carbon-coated grid. In actual use they are placed on surfaces like silicon which is very different from the grid material. To assess the state of the

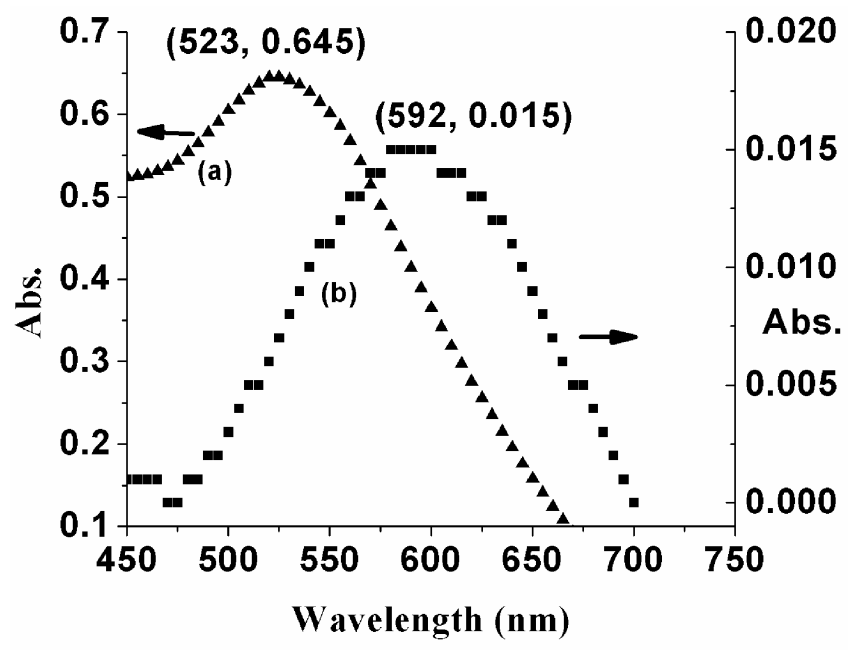

Figure 1. (a) UV-vis absorption spectrum of the prepared $\mathrm{Au}$ sol (in toluene) and (b) UV-vis absorption spectrum of the prepared $\mathrm{Au}$ nanoparticles on glass substrate. 

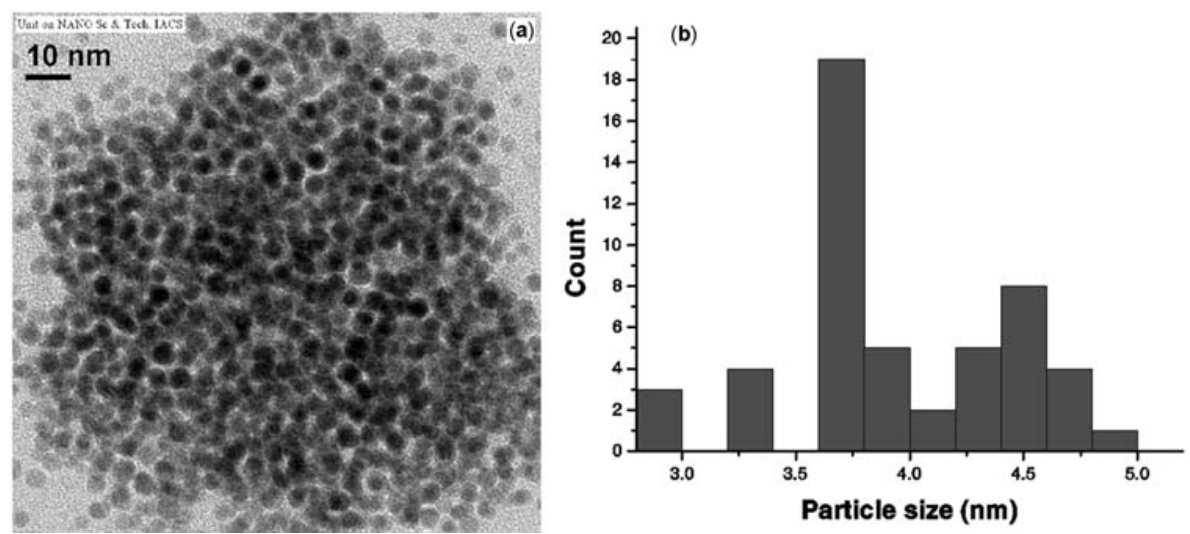

Figure 2. a. TEM image of gold nanoparticles and $\mathbf{b}$. particle size distribution from TEM data.

(a) $(255,147) \times 0.356 \mathrm{~mm}$ y. $0.2054 \mathrm{um}=0.007780 \mathrm{um}$

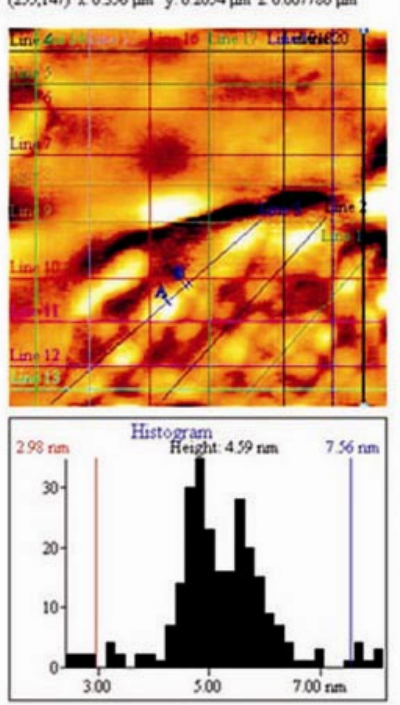

(b) 253,125$) \times 0.353 \mu \mathrm{mm}$ y.0.1788 $\mu \mathrm{m}=0.0038 \mathrm{~V}$

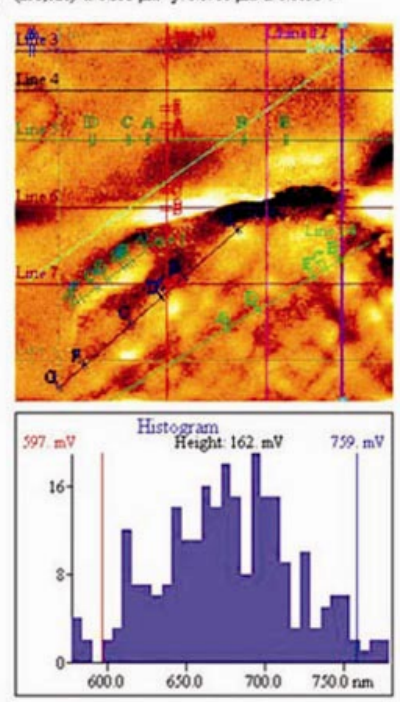

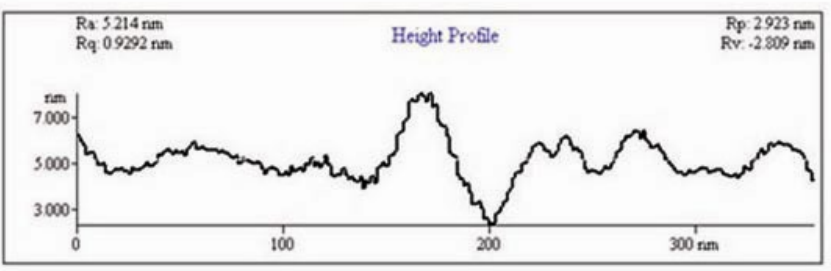
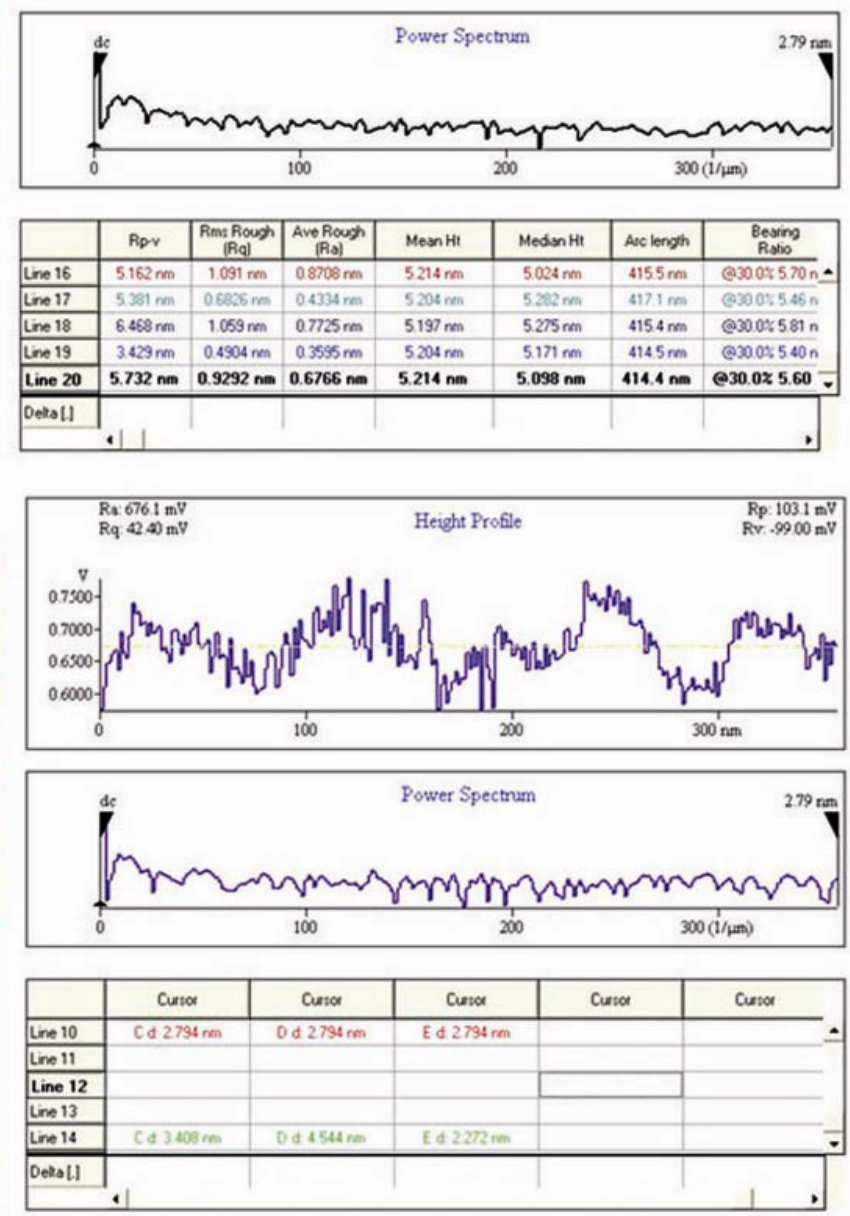

Figure 3. a. Topographical image (non-contact mode) of gold nanoparticles (with line scan) spincoated on silicon substrate $(7000 \mathrm{rpm})$. Scan size $367 \times 367 \mathrm{~nm}$ and b. phase image (non-contact mode) of gold nanoparticles (with line scan) spin-coated on silicon substrate $(7000 \mathrm{rpm}$ ). Scan size, $367 \times 367 \mathrm{~nm}$. 


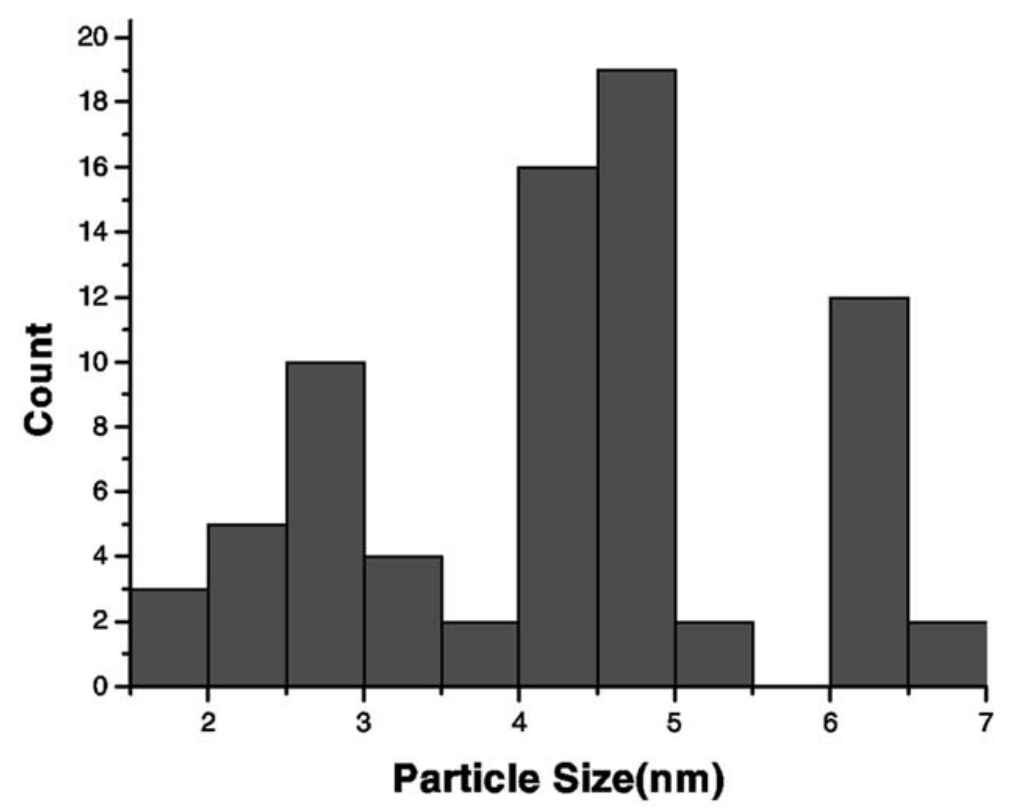

Figure 4. Particle size distribution from non-contact mode AFM data.

(a)

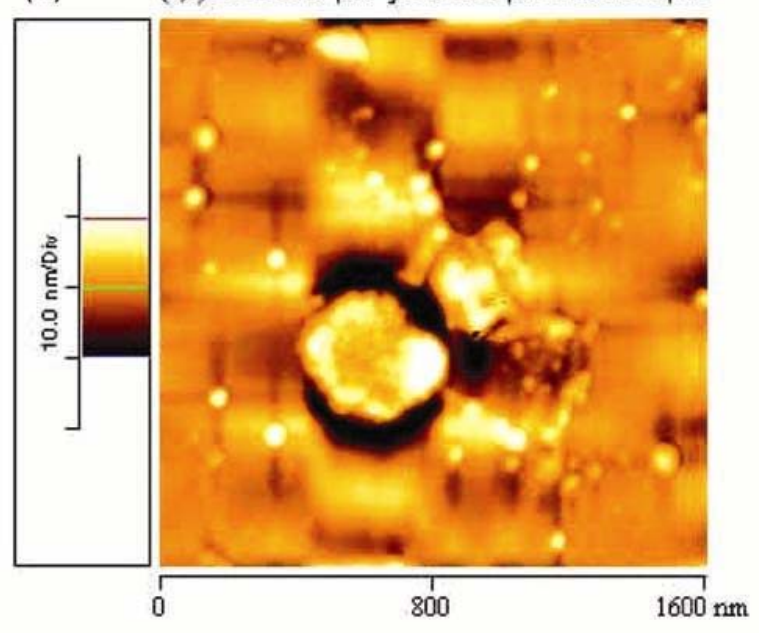

(b) (141,13) x: $0.887 \mu \mathrm{m}$ y: $0.08173 \mu \mathrm{m} z:-7.787 \mathrm{~V}$
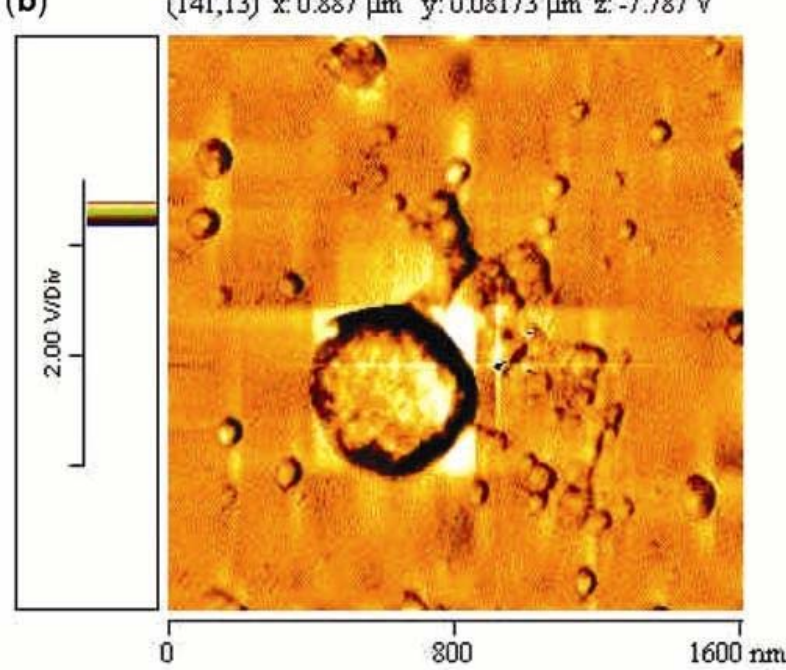

Figure 5. a. Topographical image of gold nanoparticles spin coated on silicon substrate (7000 rpm). Spherical agglomeration (size ranging from 30 to $400 \mathrm{~nm}$ ) at the middle portion of the substrate. Scan size, $1.6 \times 1.6 \mu \mathrm{m}$ and b. phase image of gold nanoparticles spin coated on silicon substrate $(7000 \mathrm{rpm})$. Spherical agglomeration (size ranging from 30 to $400 \mathrm{~nm}$ ) at the middle portion of the substrate. Scan size, $1.6 \times 1.6 \mu \mathrm{m}$.

$\mathrm{Au}$ nanoparticles we used an atomic force microscope (AFM). However, imaging of nanoparticles of such sizes is not very straightforward due to the finite size of the tip used. For this a good amount of statistics is generated from AFM line scans to find out the state of $\mathrm{Au}$ nanoparticles. The particle size was calculated from non-contact mode AFM data. AFM images were taken using CP II from Veeco (Veeco Instruments Inc., New York). Noncontact mode images were taken using a silicon tip having spring constant, $0.9 \mathrm{~N} / \mathrm{m}$ and resonance frequency,
$22 \cdot 83 \mathrm{kHz}$. The set frequency was $22 \cdot 80 \mathrm{kHz}$. Figures $3 \mathrm{a}$ and $b$ show both the non-contact mode topography and phase images (with line scan) of Au nanoparticles spin coated on silicon substrate at $7000 \mathrm{rpm}$ for $30 \mathrm{~s}$. Substrates were cleaned by well known RCA cleaning. The concentration of the solution was $2.54 \mathrm{mg} / \mathrm{ml}$. The images were taken on an island formed by assembly of such nanoparticles. Most of the islands are closer to circular shape. The average particle size calculated from AFM data is $4 \cdot 26 \pm 1 \cdot 31 \mathrm{~nm}$ (calculated from non-contact mode 
(a)

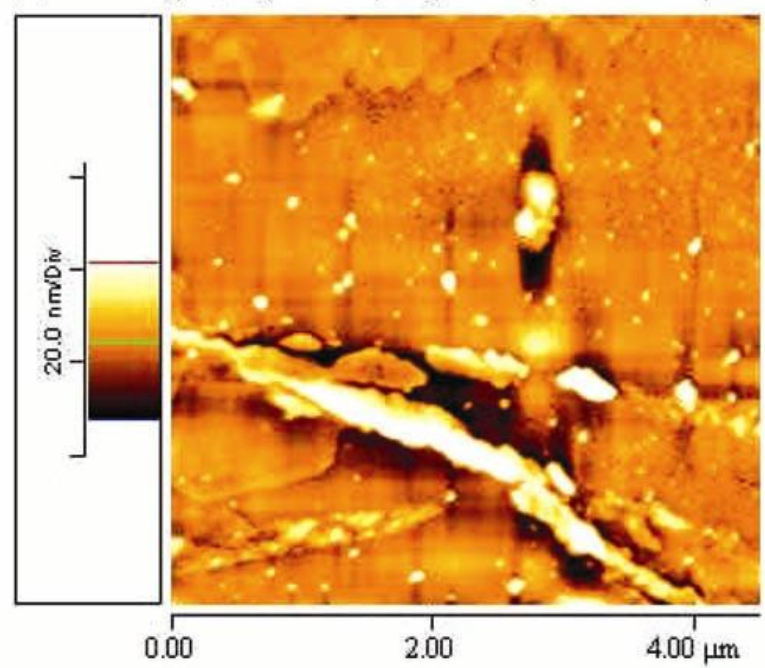

(b) (128,21U) $x: 2.23 \mu \mathrm{m}$ y: $3.691 \mu \mathrm{m} z:-9.036 \mathrm{~V}$

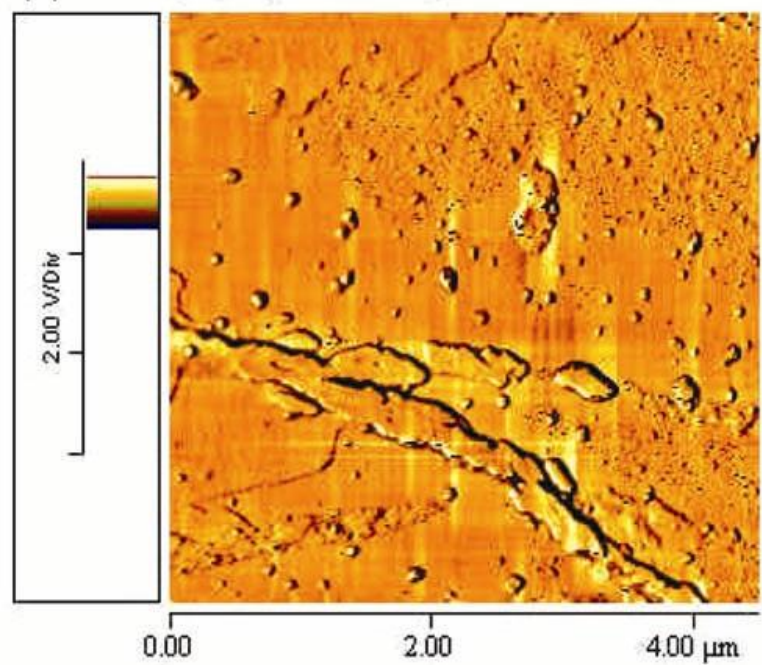

Figure 6. a. Topographical image of gold nanoparticles spin coated on silicon substrate (7000 rpm). Long structure (size, $\sim 4 \mu \mathrm{m}$ ) at the corner of the substrate. Scan size, $4.5 \times 4.5 \mu \mathrm{m}$ and $\mathbf{b}$. phase image of gold nanoparticles spin coated on silicon substrate $(7000 \mathrm{rpm})$. Long structure (size, $\sim 4 \mu \mathrm{m}$ ) at the corner of the substrate. Scan size, $4.5 \times 4.5 \mu \mathrm{m}$.

data) which is in good agreement with the TEM data shown above. The particle size was calculated from contact mode AFM (LFM) data for further confirmation and it came out to be $4 \cdot 28 \pm 1 \cdot 26 \mathrm{~nm}$. Figure 4 shows the particle size distribution from non-contact mode AFM data.

The spin coating of Au sol on the silicon substrate does not lead to one kind of structure. It has been noticed that two different types of structures were formed on silicon substrate because of spin coating at $7000 \mathrm{rpm}$. The surface of the substrate was hydrophilic. At the centre of the substrate spherical agglomeration of $\mathrm{Au}$ nanoparticles (size ranging from $30 \mathrm{~nm}$ to $400 \mathrm{~nm}$ ) were found whereas at the corner of the substrate we noticed long structures (size, $\sim 4 \mu \mathrm{m}$ ) as shown in figures $5(\mathrm{a}, \mathrm{b})$ and $6(\mathrm{a}, \mathrm{b})$, respectively. These long structures, known as 'fingers' (Schwartz and Roy 2004), are believed to be formed because of imperfect wetting behaviour during spin coating at the expanding front.

Here both the topographical and phase images are shown and it can be easily seen that the contrast is much more in phase images than topographical images. Both types of structures have the $\mathrm{Au}$ nanoparticles in similar state of assembly. However, the long types can grow up to sizes as large as $4 \mu \mathrm{m}$. The observed self-assembly depends on hydrophilic nature of the substrate. By making the substrates hydrophobic (by HF etching) does not lead to such self-assembly.

Spin coating is a simple and effective technique in which to deposit thin, uniform films across planar substrates. The film solution generally consists of volatile solvents in solution with effectively non-volatile solutes. This solution is dispersed onto a planar surface that is then accelerated to a predetermined rotation rate. Evapo- ration of the volatile constituents occurs from the top surface of the solution, while simultaneously the rotation (centrifugal force) forces the fluid to flow uniformly outward. Typically, fluid flow considerations dominate the early part of spinning while solvent evaporation controls the behaviour at later stages. The thinning rate of solution which is attributed to viscous flow varies as the cube of solution thickness (and the square of spin speed), so this dominates the early stage of spinning. The thinning rate of solution contributed by solvent evaporation is independent of solution thickness because it occurs only at the top surface and is limited by diffusion of the evaporated solvent molecules through a laminar vapour boundary layer above the solution/air interface.

\section{Conclusions}

In this work, a systematic study on the large pattern formation of uncapped Au nanoparticles by spin coating was done. The pattern, as stated before has been formed in two stages, first, self-assembly of Au nanoparticles to $\mu \mathrm{m}$ size islands; second, formation of larger arrays of the islands. The pattern formation depends on the hydrophilic nature of the silicon substrate. In the process of spincoating, the centrifugal forces cause the liquid to drain radially off the edge of the substrate until solvent evaporation leaves a solute-rich liquid. The solute-rich phase is too viscous to flow readily and ultimately forms large concentric arrays of liquid droplets. The droplets contain the Au nanoparticles, which then grow into an array within the droplets. The pattern thus obtained contains arrays of islands $(\sim 30-400 \mathrm{~nm})$ each of which is an array of $\mathrm{Au}$ nanoparticles. 


\section{Acknowledgements}

The authors are thankful to the Department of Science and Technology, New Delhi, for financial support. They also want to thank Unit for Nanoscience, Indian Association for the Cultivation of Science, Kolkata, for TEM use.

\section{References}

Brust M, Walker M, Bethell D, Schiffrin D J and Whyman R 1994 J. Chem. Soc., Chem. Commun. 801

Choi G S, Cho Y S, Son K H and Kim D J 2003 Microelectron. Eng. 6677

Cho Y S, Choi G S, Hong S Y and Kim D 2002 J. Cryst. Growth 243224

Drake P and Youngs I 2002 Mater. Sci. Technol. 18772

Green R J, Frazier R A, Shakesheff K M, Davies M C, Roberts C J and Tendler S J B 2000 Biomaterials 211823

Hong Y K, Kim H, Lee G and Kim W 2002 Appl. Phys. Lett. 80 844
Kodama H, Momose S, Ihara N, Uzumaki T and Tanaka A 2003 Appl. Phys. Lett. 835253

Lin J, Zhou W and O'Connor C J 2001 J. Mater. Lett. 49 282

Liu F K, Chang Y C, Ko F H, Chu T C and Dai B T 2003 Microelectron. Eng. 67/68 702

Martin J E, Wilcoxon J P, Odinek J and Provencio P $2000 \mathrm{~J}$. Phys. Chem. B 1049475

Maye Mathew M, Zheng Wenxia, Leibowitz Frank L, Ly Nam $\mathrm{K}$ and Zhong Chuan-Jian 2000 Langmuir 16490

Schwartz L W and Roy R V 2004 Phys. Fluids 16569

Spatz J P, Roescher A and Moleller M 1996 Adv. Mater. 8 337

Templeton Allen C, Peter Wuelfing W and Murray Royce W 2000 Acc. Chem. Res. 3327

Underwood Sylvia and Mulvaney Paul 1994 Langmuir 10 3427

Weare W W, Reed S M, Warner M G and Hutchison J E 2000 J. Am. Chem. Soc. 12212890

Zhou Y, Itoh H, Uemura T, Naka K and Chujo Y 2002 Langmuir 18277 